\title{
Fully interconnected, two-dimensional neural arrays using wavelength-multiplexed volume holograms
}

\author{
Robert T. Weverka and Kelvin Wagner \\ Department of Electrical and Computer Engineering and National Science Foundation Engineering Research Center for Optoelectronic \\ Computing Systems, University of Colorado, Boulder, Colorado 80309-0425
}

\author{
Mark Saffman
}

Department of Physics and Joint Institute for Laboratory Astrophysics, University of Colorado, Boulder, Colorado 80309-0440

Received August 27, 1990

\begin{abstract}
We present a compact method to provide independent weighted interconnections between every pixel in a twodimensional input array and every pixel in a two-dimensional output array. The two input dimensions and two output dimensions are connected by a four-dimensional weight matrix consisting of wavelength-multiplexed volume holograms that use cryogenic spectral hole burning in a single holographic element.
\end{abstract}

Neural networks typically require weighted global interconnections between arrays of simple soft threshold units. Optics provides a natural two-dimensional (2-D) representation of high-resolution images for parallel processing applications. However, the global interconnection of images with $1000 \times 1000$ pixels requires a four-dimensional interconnection matrix with $10^{12}$ degrees of freedom. Although numerous schemes for optical interconnections have been advanced, a technique that globally interconnects dense 2-D diffraction-limited images has not yet been suggested. Several authors have proposed the use of 2-D spatially multiplexed arrays of holograms to interconnect 2-D images. ${ }^{1-6}$ Difficulties arise with this approach when it is applied to high-resolution images, including large planar holograms, spatial light modulators, detectors and lenses, and the resulting large system volume. An alternative approach to implementing holographic neural networks uses volume holograms. However, the Bragg degeneracy of volume gratings restricts the interconnection topology to relatively sparse fractal grids. ${ }^{7,8}$

Fourier volume holograms provide a limited number of degrees of freedom due to Bragg degeneracy. The $N^{3}$ degrees of freedom in a volume holographic medium can be used to connect at most $N$-to- $N^{2}$ pixels or $N^{3 / 2}$-to- $N^{3 / 2}$ pixels, where $N$ is the number of optically resolvable spots in one dimension. ${ }^{7-9}$ The interconnection density can be increased by using spectral hole-burning materials and the wavelength as a multiplex parameter. The volume holographic gratings written in spectral hole-burning materials at separate wavelengths are completely independent, if separated by a sufficient wavelength interval. $N$ wavelengths can provide an additional factor of $N$ interconnects in order to give full interconnection between $N^{2}$ inputs and $N^{2}$ outputs with $N^{4}$ degrees of freedom. We propose three methods for combining fully connected sparse fractal arrays with a regular arrangement of wavelength multiplexing and spectral filtering to pro- vide full $N^{2}$ fan out and $N^{2}$ fan in to each pixel (i.e., fully interconnected 2-D arrays).

The spectrally specific gratings necessary to achieve $N^{4}$ independent interconnects can be written using persistent spectral hole burning. By cooling a suitable inhomogeneously broadened medium to cryogenic temperatures, the homogeneous linewidth becomes narrow, which enables a large number of independent holograms to be wavelength multiplexed. The multiplex factor is limited by the ratio of the inhomogeneous to homogeneous linewidths of the relevant electronic transition. Materials have been developed that have the potential to multiplex of the order of 10,000 wavelengths. ${ }^{10}$ Holographic detection of the spectral holes implies that both absorption and refractive index changes contribute to the grating strength, which results in a broader effective linewidth. ${ }^{11}$ This will reduce the number of independent gratings that can be stored to of the order of 1000 , which should be sufficient for the envisaged application.

The general approach, shown in Fig. 1, involves wavelength multiplexing in the dimension of Bragg degeneracy. The arrangement of input and output pixels, connected by the first few wavelengths of the nine wavelength-multiplexed $9 \times 9$ to $9 \times 9$ interconnect, is shown in Fig. 2. Consider the connections between input array A and output array B. The first wavelength, $\lambda_{1}$, only illuminates the first row in Fig. 2(a). Each of the different pixels in the first row of inputs is Bragg matched to its own unique set of $N^{2}$ gratings, giving $N^{3}$ total gratings at $\lambda_{1}$. While Bragg selectivity allows distinct pixels in the same row to couple through separate gratings, there is Bragg degeneracy along the column direction, which is eliminated by using a distinct wavelength for each row. At a second wavelength, illuminating the second row, an entirely new set of spectral hole-burned gratings is written between the second row of inputs and the 2-D array of outputs [Fig. 2(b)]. Each of the successive rows is illuminated with a new wavelength that inter- 


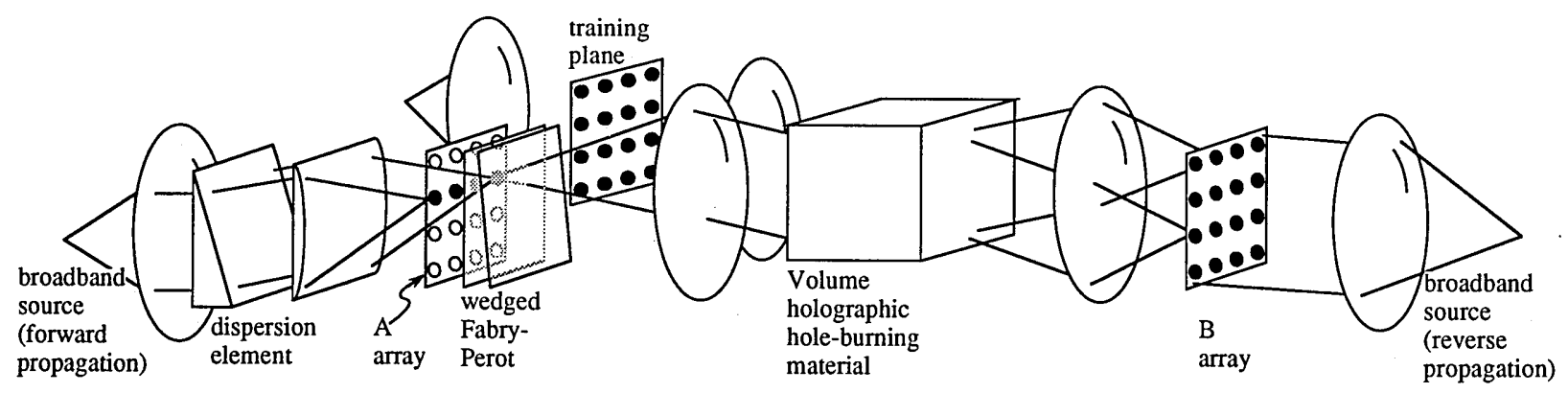

Fig. 1. Wavelength-multiplexed volume holographic interconnect. Light from the broadband source at the left is dispersed to illuminate array A with an optical frequency that increases linearly along the vertical direction. Array A modulates this light, which is spectrally matched to the passband of the wedged Fabry-Perot étalon. The light from each pixel is collimated by the lens and interconnected by the hologram to array B for detection. When the system is used in the reverse direction, each pixel at array $B$ modulates the entire spectrum. The Bragg degenerate interconnections in the column direction are filtered out by the wedged Fabry-Perot étalon. The light that passes the Fabry-Perot étalon is detected at the neural outputs in array A. The training plane is used in conjunction with the input A to expose the spectral hole-burning holograms.

A

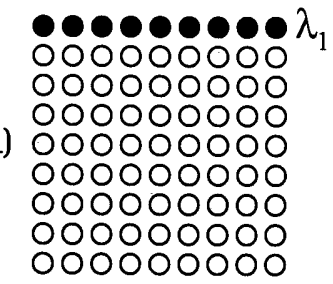

000000000

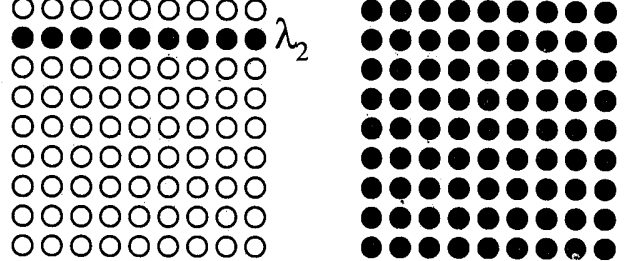

Fig. 2. Interconnect topology showing the connections for the first three wavelengths out of nine in a $9 \times 9$ to $9 \times 9$ interconnection scheme. (a) shows the pattern used by $\lambda_{1}$, and (b) shows the pattern used by $\lambda_{2}$.

acts with different volume holograms, which gives us independent interconnection strengths from each pixel in the input, A, to each pixel in the output, B.

Interconnections may be realized in the opposite direction as well, which may be important for bidirectional associative memories ${ }^{12}$ or for bidirectional learning systems such as backpropagation..$^{13}$ Figure 1 shows a broadband source illuminating all the pixels for reverse propagation. At a single illumination wavelength, the Bragg selectivity gives us distinct connections from all the inputs, $B$, to each of the elements of a single row in the output array A. However, the Bragg degeneracy connects these inputs with the same weights to the other output rows as well. The wedged Fabry-Perot étalon ${ }^{14}$ can then be used to select a single wavelength at each output row and eliminate Bragg degenerate diffractions. With the Fabry-Perot étalon set to pass the first wavelength $\lambda_{1}$ at the top row and successive wavelengths at the succeeding rows, the wavelength-multiplexed holograms that provide these reverse-direction interconnections are the same volume holograms as those used to perform the forward interconnections described above.
A more symmetric 2-D to 2-D wavelength-multiplexed interconnection can be based on an $N^{3 / 2}$ coarse array to an $N^{3 / 2}$ fine array fractal interconnect. 7,8 Figure 3 shows the interconnections for the first three of nine wavelengths in a $9 \times 9$ to $9 \times 9$ interconnection scheme. Each of the first three rows is illuminated by the first three wavelengths. The light at $\lambda_{1}$ in Fig. 3(a) is diffracted from each of the inputs in the first three rows to each of the outputs in the first, fourth, and seventh output rows. This constitutes our desired $N^{3}$ interconnections at $\lambda_{1}$. The Bragg degeneracy diffracts this $\lambda_{1}$ light to the output rows denoted by the hatched circles in Fig. 3 as well. A spectral filter is required to reject the $\lambda_{1}$ light at these positions. Figure 3 shows the connections at $\lambda_{2}$ and $\lambda_{3}$ by the diagonal and vertical hatching. These $N^{1 / 2}$ wavelengths provide all the interconnections from the first $N^{1 / 2}$ input rows to the entire $N \times N$ output plane. The second set of $N^{1 / 2}$ input plane rows (row 4 through 6 in our $N=9$ example) are connected to the outputs by the second set of $N^{1 / 2}$ wavelengths in the same fashion, and so on for successive groups of $N^{1 / 2}$ wavelengths. The wavelength-selective filter that provides the rejection of the degenerate Bragg diffractions has a periodic multiline bandpass function at any one position, and the bandpass wavelengths increase linearly with vertical position along the output. This is the periodic bandpass of a wedged Fabry-Perot étalon with a larger mirror spacing.

The interconnection schemes outlined above have both coherent and incoherent fan in. Fan in from each individual wavelength is performed coherently,
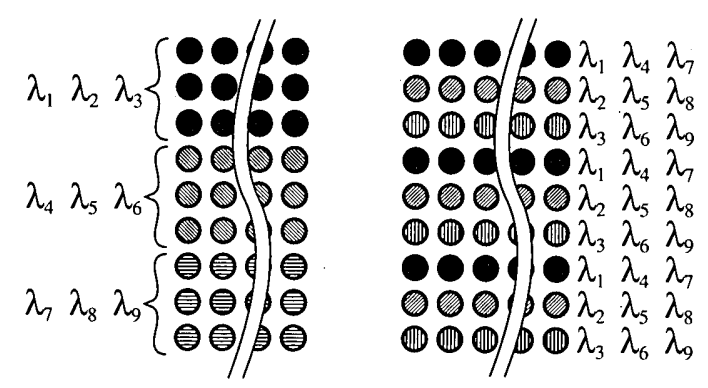

Fig. 3. Symmetric interconnect showing the first group of $N^{1 / 2}$ wavelengths. 
while multiple wavelengths are combined incoherently in some of the schemes. The coherent fan in through gratings with controlled phase can provide for both sum and difference terms in the weighted interconnects. The multiwavelength fan in is incoherent and can provide only positive weights in the summations of each of the single-wavelength partial sums. The intensity at the $i j$ th output pixel is given by the coherent-incoherent summation,

$$
I_{i j}=\frac{1}{N^{m}} \sum_{p=0}^{N^{1-m}-1}\left|\sum_{k=p N^{m}+1}^{(p+1) N^{m}} \sum_{l=1}^{N} W_{i j k l} a_{k l}\right|^{2},
$$

where $N$ is the number of optically resolvable spots in one dimension, $m+1$ is the degree of coherent fan in, 0 $<m<1, a_{k l}$ is the optical modulation at the inputplane spatial light modulator, $l$ is the index ranging over the input pixels of the same optical wavelength and in the same row, $k$ ranges over the rows, $p$ ranges over the wavelengths that fan in incoherently, $W_{i j k l}$ is the four-dimensional interconnection weight matrix, and the wavelength is given by $\lambda=\lambda_{0}+\Delta \lambda\left(p N^{m}+i\right.$ $\bmod N^{m}$ ). When it is necessary to provide the capability for either positive or negative weights for all the neural interconnections, then the propagation from $B$ to A in Fig. 2 must be used, since it provides completely coherent summations.

The optical throughput is limited by the achievable diffraction efficiency in a spectral hole-burning material. In addition, the optical efficiency in the interconnection schemes is further reduced by the optical filters required in the second and third schemes. The optical efficiency, $1 / N^{m}$, decreases with increasing coherent fan in, $N^{m+1}$. The first scheme (connections from A to B in Fig. 2) has $m=0$ and a coherent fan in of $N$ owing to summations within a row and has no light loss since it requires no optical filters. In the reverse propagation direction, for which $m=1$, a filter is required at the output plane that passes a factor of $1 / N$ of the incident light in order to reject the Bragg degenerate interconnections. However, in this reverse direction it has a fully coherent fan in of $N^{2}$. The symmetric scheme has the optical filter passing $1 / N^{1 / 2}$ of the incident light and a coherent fan in of $N^{3 / 2}$. We suspect that any wavelength-multiplexed volume holographic interconnect scheme will have this same trade-off between an optical loss due to wavelength filtering of $1 / N^{m}$ and a coherent fan in of $N^{1+m}$, unless coherent wavelength fan in using mode-locked pulsed sources and temporally gated detectors is employed.

The key to utilizing the large numbers of degrees of freedom available in a spectral hole-burning volume holographic interconnect is the ability to write the desired weights through a sequence of exposures controlled by a learning algorithm or an exposure scheduling sequence. ${ }^{13,15}$ Neural learning usually requires the adaptive recording of a series of outer-product image associations that are used to modify the $N^{4}$ synaptic weights. The training plane illustrated in Fig. 1 is an image of the B plane and is used for recording the desired outer product holograms. The images to be holographiclly associated are used to modulate the dispersed light illuminating the A plane and the broadband illumination of the training plane.
At a given wavelength, a row of pixels in $\mathrm{A}$ interferes with every pixel in $\mathrm{B}$, resulting in $N^{3}$ gratings, and the simultaneous exposure by $N$ wavelengths permits the modification of all $N^{4}$ gratings on each exposure. Successive exposures do not erase previous exposures, but each uses up a fraction of the available dynamic range. This limits the average diffraction efficiency of each grating to the saturation diffraction efficiency divided by the square of the number of exposures. ${ }^{8}$ Both an increase and a decrease of interconnection strengths are required for learning to converge. Although coherent erasure should be possible in these materials, it may result in saturation unless grating persistence can be controlled. In addition, an extremely large number of pattern presentations and learning cycles may be necessary. These issues raise different material requirements than typical spectral hole-burning applications such as data storage and may necessitate a search for new materials before a practical implementation of a learning system can be expected.

In summary, we have presented wavelength-multiplexing schemes for interconnecting fully populated 2-D arrays using spectral hole-burning holography. These schemes may be used to implement optical neural networks with $N^{2}$ inputs and $N^{2}$ outputs, where $N$ is the number of optically resolvable spots in one dimension. We have examined their efficiencies and degree of coherent fan in and pointed out the trade-off between the two, and we have discussed some of the issues involved in programming these interconnections.

Note added in proof: A related scheme was proposed recently in Ref. 16.

\section{References}

1. D. Psaltis and N. Farhat, Opt. Lett. 10, 98 (1985).

2. H. J. Caulfield, Appl. Opt. 26, 4039 (1987).

3. J.-S. Jang, S.-W. Jung, S.-Y. Lee, and S.-Y. Shin, Opt. Lett. 13, 248 (1988).

4. S. H. Song and S. S. Lee, Appl. Opt. 27, 3149 (1988).

5. M. Ishikawa, N. Mukohzaka, H. Toyoda, and Y. Suzuki, Appl. Opt. 28, 291 (1989).

6. G. Y. Sirat, A. D. Maruani, and R. C. Chevallier, Appl. Opt. 28, 1429 (1989).

7. H. Lee, X.-G. Gu, and D. Psaltis, J. Appl. Phys. 65, 2191 (1989).

8. D. Brady, Ph.D. dissertation (California Institute of Technology, Pasadena, Calif., 1989).

9. D. Psaltis, C. H. Park, and J. Hong, Neural Networks 1, 149 (1988).

10. F. M. Schellenberg, W. Lenth, and G. C. Bjorklund, Appl. Opt. 25, 3207 (1986).

11. A. J. Meixner, A. Renn, and U. P. Wild, J. Chem. Phys. 91, 6728 (1989).

12. C. C. Guest and R. Tekolste, Appl. Opt. 26, 5055 (1987).

13. K. Wagner and D. Psaltis, Appl. Opt. 26, 5061 (1987).

14. M. Born and E. Wolf, Principles of Optics (Pergamon, New York, 1986), p. 351.

15. D. Brady, D. Psaltis, and K. Wagner, Appl. Opt. 27, 1752 (1988).

16. P. Henshaw, in Digest of Optical Society of America Annual Meeting (Optical Society of America, Washington, D.C., 1990), p. 57. 